\title{
High-connectivity quantum processor nodes using single-ion qubits in rare-earth-ion-doped crystals
}

\author{
Adam Kinos $\odot,{ }^{1, *}$ Lars Rippe, ${ }^{1}$ Diana Serrano $\odot,{ }^{2}$ Andreas Walther,${ }^{1}$ and Stefan Kröll ${ }^{1}$ \\ ${ }^{1}$ Department of Physics, Lund University, P.O. Box 118, SE-22100 Lund, Sweden \\ ${ }^{2}$ Chimie Paris Tech, PSL University, CNRS, Institut de Recherche de Chimie Paris, Paris, 75005, France
}

(Received 18 November 2021; revised 1 February 2022; accepted 22 February 2022; published 4 March 2022)

\begin{abstract}
We present two protocols for constructing quantum processor nodes in randomly doped rare-earth-ion crystals and analyze their properties. By varying the doping concentration and the accessible laser tunability, the processor nodes can contain anywhere from only a few tens to almost 1000 qubits. Furthermore, the average number of qubits each qubit can interact with, denoted by the connectivity, can be partly tailored to lie between just a few and roughly 100 . We also study how a limited tunability of the laser affects the results and conclude that a tuning range of $100 \mathrm{GHz}$ limits the results to roughly 100 qubits with around 50 connections per qubit on average. In order to construct an even larger processor, the vision is that several of these quantum processor nodes should be connected to each other in a multinode architecture via, e.g., optical interfaces or flying qubits in the form of light. Our results are encouraging for establishing the rare-earth-ion-based systems as a quantum computing platform with strong potential and can serve to focus the efforts within the field.
\end{abstract}

DOI: 10.1103/PhysRevA.105.032603

\section{INTRODUCTION}

In the field of quantum information, rare-earth-ion-doped crystals have been used in quantum memories [1-12], conversion between optical and microwave signals [13-15], and quantum computing [16-26]; the latter topic is here investigated through simulations. In order to focus the research community on the task of constructing a rare-earth quantum computer, a road map has been developed [27]. There the qubits are envisioned to be single ${ }^{153} \mathrm{Eu}$ ions randomly doped at the few percent level into a $\mathrm{Y}_{2} \mathrm{SiO}_{5}$ crystal (or nanocrystal or thin film). The $|0\rangle$ and $|1\rangle$ states of a qubit are encoded in two ground states of a single Eu ion, and previous work has indicated that single- and two-qubit gate operations with low errors are possible [28].

Gate operations are performed via an excited state by using optical pulses. Furthermore, different qubits occupy different frequency channels, and thus frequency selection can be used to control different qubits. This is possible since the qubit transitions have kilohertz linewidths, and due to the random nature of the doped crystal, the surrounding of each dopant is slightly different and leads to an inhomogeneous broadening in their transition frequencies. This broadening depends on the doping concentration and can be in the order of $100 \mathrm{GHz}$ [29]. Thus the laser used to control a qubit only interacts with a small subset of all dopants. However, due to the high doping con-

\footnotetext{
*adam.kinos@fysik.lth.se
}

Published by the American Physical Society under the terms of the Creative Commons Attribution 4.0 International license. Further distribution of this work must maintain attribution to the author(s) and the published article's title, journal citation, and DOI. Funded by Bibsam. centration there could still be several thousands of nonqubit $\mathrm{Eu}$ ions within the focus size and bandwidth of the laser. If such nonqubit ions are excited, dipole-dipole interactions with the qubit ion can cause instantaneous spectral diffusion (ISD), which can increase the error of gate operations performed on the qubit. To reduce the risk of ISD, optical pumping techniques [19,30-32] and more complicated procedures [21] can be utilized. Previous work has investigated the effects of ISD on single-qubit gate operations and concluded that operations with low errors are still possible in the vast majority of cases [33].

The present work does not make any assumptions on how the state of a single-ion Eu qubit is read out, but directly reading out the state of the qubit is very difficult due to the high doping concentration. However, a possible method is to use dedicated readout ions [21,23,27], which are codoped with the qubit dopants but at a much lower concentration. Single-ion readout of such dedicated readout ions is deemed feasible due to the recent progress in single-ion detection [34-39]. State selective readout of a Eu qubit is then possible by using the dipole-dipole blockade mechanism between the Eu qubit and a dedicated readout ion to either enable or disable the fluorescence of the readout ion.

The spectral addressing of qubits makes it possible to pack the ions at nanometer spacing in three dimensions. This allows for very high qubit densities. In addition, the dense packing allows for strong dipole-dipole interactions between qubits [18,19,40-42]. However, the number of potential qubits in these systems and their connectivity has previously not been carefully investigated, and this is a central question for assessing the potential of rare-earth systems as a quantum computing platform.

In this work we examine quantum processor nodes in randomly doped rare-earth-ion crystals. There are numerous ways to select the ions that should be used as qubits, and 


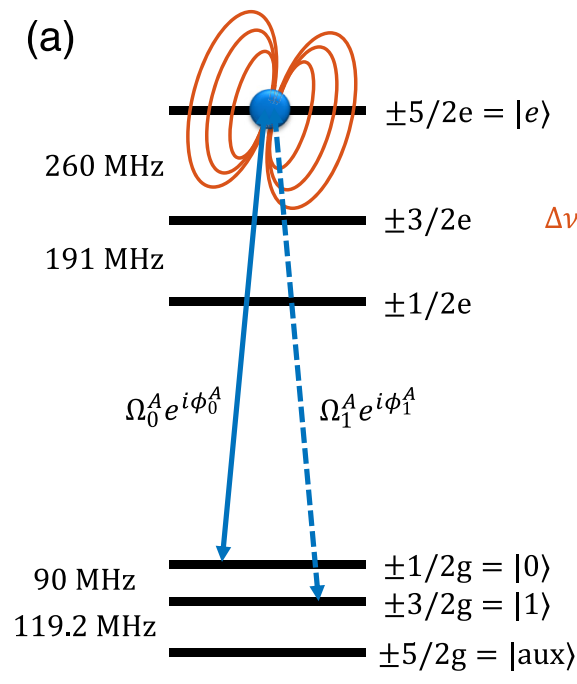

Qubit 1 (b)

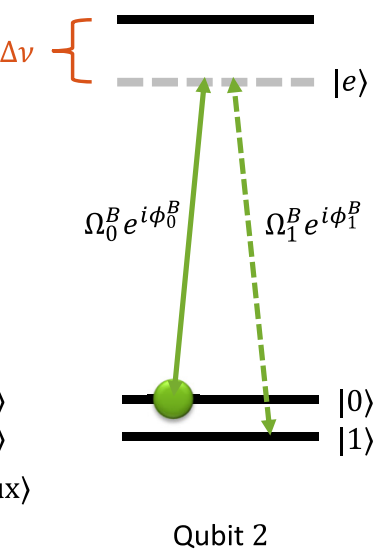

(c)

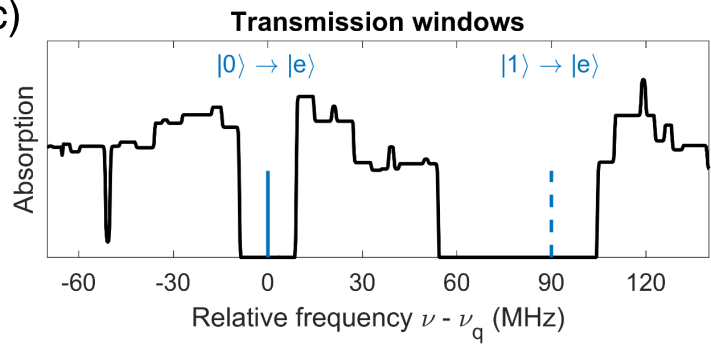

(d)

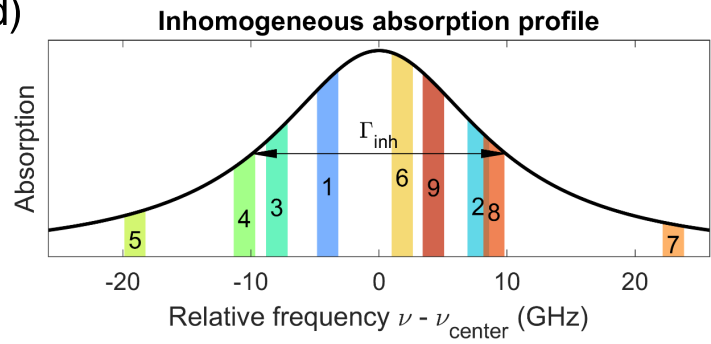

FIG. 1. (a) Shows the energy-level structure for a qubit including the two-color pulses used to control it. The two fields driving the $|0\rangle \rightarrow|e\rangle$ and $|1\rangle \rightarrow|e\rangle$ qubit transitions are shown as solid and dashed lines, respectively. (b) Shows only the relevant qubit levels for a second qubit whose optical transitions are detuned from the first qubit. When either of the qubits are excited, the other experiences a frequency shift, $\Delta v$, of its optical transitions due to a dipole-dipole interaction. The strength of this interaction scales as $1 /|\boldsymbol{r}|^{3}$, where $|\boldsymbol{r}|$ is the distance between the two qubits [43]. (c) In order to minimize the effect of ISD, one can empty the frequency regions surrounding the two qubit transitions using, e.g., optical pumping, such that there are no absorbing ions in those regions. Here such transmission windows with widths of roughly $18 \mathrm{MHz}$ and $50 \mathrm{MHz}$, respectively, are shown as a function of the relative frequency $v-v_{q}$, where $v_{q}$ is the $|0\rangle \rightarrow|e\rangle$ transition frequency of the qubit with index $q$. (d) The inhomogeneous absorption profile of the optical transitions is shown, including the FWHM, $\Gamma_{\text {inh }}$, determined by Eq. (2). Here we use $c_{\text {total }}=1 \%$ as an example. We also show a schematic representation of nine qubits with indices $1 \rightarrow 9$ and different transition frequencies $v_{q}$. The colored regions show the reserved frequency interval surrounding each qubit, and other qubits may not have their $v_{q}$ transition frequency within this interval. Note, however, that as long as the $v_{q}$ transition frequencies are outside other colored regions, those regions may themselves partly overlap. This can be seen for the qubits with indices 2 and 8 .

depending on which way is used the connectivity of the processor node can vary drastically. Here we focus on two extreme protocols for selecting the qubit ions. Using the first protocol results in qubits that are densely distributed in space and have high connectivity. In contrast, using the second protocol yields qubits that are sparsely distributed and have low connectivity. We conclude that both the number of qubits and the connectivity between qubits can be partly tailored by modifying these protocols or changing physical parameters, such as doping concentration, and technological parameters, such as the available laser tunability. The quantum processor nodes in this article reach qubit numbers between a few tens and 1000 , and the average number of connections per qubit ranges from a few connections up to 100 .

We now provide an overview of the sections and content of the paper. Section II provides all necessary information about how the processor node is constructed. Following this, Sec. III investigates the number of qubits and their connectivity for processors created using different protocols and/or doping concentrations, and we also discuss the effect of a limited laser tunability. Finally, this work is concluded in Sec. IV.

\section{THE CONSTRUCTION OF QUANTUM PROCESSOR NODES}

\section{A. Material overview}

In this work we investigate a quantum processor node using

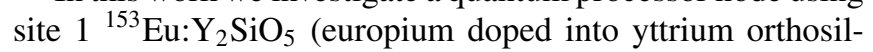

icate), whose relevant properties can be found in Fig. 1(a). Single-qubit (SQ) gate operations are performed using two, two-color pulses resonant with the $|0\rangle \rightarrow|e\rangle$ and $|1\rangle \rightarrow|e\rangle$ qubit transitions following the procedure outlined and motivated in Ref. [28]. The average SQ gate error is estimated to be $3.4 \times 10^{-4}$ [28], and even if instantaneous spectral diffusion (ISD) is accounted for, the error is in the same order of magnitude for the vast majority of qubits [33]. The effect of ISD is minimized by using spectral hole burning techniques [19,30-32] to isolate the qubit ions in frequency space by creating semipermanent transmission windows in the inhomogeneously broadened rare-earth-ion ensemble, see Fig. 1(c).

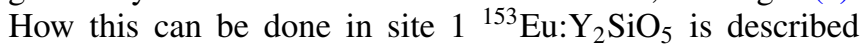
further in Ref. [33].

Two-qubit (TQ) gate operations are performed using a dipole-dipole interaction which allows different qubits to interact if they are sufficiently close in space. The dipole-dipole interaction is explained in Figs. 1(a) and 1(b) and scales as $1 /|\boldsymbol{r}|^{3}$, where $|\boldsymbol{r}|$ is the distance between the two qubits [43]. Two different types of TQ gates are used: the blockade gate, which requires dipole-dipole shifts larger than the pulse bandwidth so that if one qubit is excited the other qubit is shifted out of resonance with its control pulses, and the interaction gate, which takes advantage of the exact dipole-dipole shift to, e.g., add a phase to a specific TQ state [28]. When the dipole-dipole shift, $\Delta v$, between two qubits is sufficiently strong, $|\Delta \nu| \geqslant 7 \mathrm{MHz}$, the TQ blockade gate is used, whereas the TQ interaction gate is used when the shift is less than 
this, $0.1 \mathrm{MHz} \leqslant|\Delta v|<7 \mathrm{MHz}$. The average TQ gate errors are estimated to lie in the range of $5 \times 10^{-4} \rightarrow 3 \times 10^{-3}$, depending on the exact dipole-dipole shift; for more information see Ref. [28]. Note that these error rates lie beneath the threshold for error correction using surface codes $[44,45]$ and at the border of the threshold for CSS codes [46].

Although the SQ and TQ gates are not directly used in the simulations presented in this work, they do affect the properties of the processor. If one uses TQ gates that work for small shifts and thus large separations $|\boldsymbol{r}|$ between qubits, the resulting processor has a larger connectivity compared to if one uses TQ gates that only work for large shifts.

Furthermore, the gate parameters also affect how large frequency range each qubit must reserve in order for other qubits not to cause significant additional errors, e.g., when gate operations are performed on those other qubits. Reference [28] investigated how the control pulses of one qubit affect the state of another qubit as a function of the frequency difference between the $|0\rangle \rightarrow|e\rangle$ transitions of the two qubits and concluded that a minimum detuning of roughly $\pm 0.6 \mathrm{GHz}$ was required. However, when the effect of ISD is minimized by creating transmission windows for each qubit as described above, the minimum detuning is even larger. In Appendix A we calculate the frequency detunings that must be reserved for each qubit to allow for the creation of such transmission windows and find that it covers roughly $1.7 \mathrm{GHz}$, meaning that another qubit cannot have its $|0\rangle \rightarrow|e\rangle$ transition in that interval. However, since the reserved frequency regions for different qubits can overlap as long as the $|0\rangle \rightarrow$ $|e\rangle$ transition frequency of a qubit does not enter into another region, one can, in the best case when the qubits are packed as tightly as possible in frequency space, fit one qubit per roughly $1.7 / 2 \mathrm{GHz}=0.85 \mathrm{GHz}$. A schematic view of how the qubits reserve parts of the inhomogeneous absorption profile can be seen in Fig. 1(d).

\section{B. Crystal realization}

In order to estimate the number of qubits and their connectivity for a quantum processor node we create random realizations of a crystal. Using the structure of the yttrium orthosilicate $\left(\mathrm{Y}_{2} \mathrm{SiO}_{5}\right)$ host crystal [47-49], we randomly replace a fraction, $c_{\text {total }}$, of the yttrium ions with the ${ }^{153} \mathrm{Eu}$ dopants. We assume that half of the dopants occupy site 1, which we investigate here, and thus only half of the total number of dopants can form qubits in the processor. More detailed information about the doping procedure can be found in Ref. [33].

After this random placement we know the spatial position as well as the relative direction of the static electric dipole moment difference, $|\Delta \boldsymbol{\mu}|=7.6 \times 10^{-32} \mathrm{C} \mathrm{m}$ [50], for all dopants. This allows us to calculate the dipole-dipole shift, $\Delta v$, between any two dopants $[33,43]$ :

$$
\begin{aligned}
\Delta v & =\frac{k}{|\boldsymbol{r}|^{3}}\left[\Delta \boldsymbol{\mu}_{A} \cdot \Delta \boldsymbol{\mu}_{B}-3\left(\Delta \boldsymbol{\mu}_{A} \cdot \hat{\boldsymbol{r}}\right)\left(\hat{\boldsymbol{r}} \cdot \Delta \boldsymbol{\mu}_{B}\right)\right] \\
k & =\frac{(\epsilon(0)+2)^{2}}{9 \epsilon(0)} \frac{1}{4 \pi \varepsilon_{0} h},
\end{aligned}
$$

where $\boldsymbol{r}$ is the spatial vector pointing from ion $B$ to ion $A$, and $\hat{\boldsymbol{r}}$ is the normalized spatial vector. The first term in the constant $k$ is a local field correction due to the crystal [51], where the dielectric constant for DC fields, $\epsilon(0)$, is equal to 11 for the case of $\mathrm{Y}_{2} \mathrm{SiO}_{5}$ [52,53]. $\varepsilon_{0}$ is the vacuum permittivity and $h$ is Planck's constant.

Since the surrounding of each dopant is slightly different, the absorption of all dopants form an inhomogeneous absorption profile, see Fig. 1(d). The profile is best described by a Lorentzian line shape [29], which is consistent with an inhomogeneous broadening that is caused by point defects in the crystal $[29,54]$. Note, however, that the real distribution of ion frequencies would eventually deviate from the smooth decay of a Lorentzian shape, since satellite lines would instead form $[55,56]$ due to the discrete placement of the dopants in the crystal. Furthermore, dopants situated close in space almost experience the same surrounding, and there might therefore exist a correlation between ions having similar transition frequencies and being spatially close to each other. However, even for dopants that form satellite lines and thus are very close in space, this correlation is relatively small [56]. Since most dopants are further away from each other than this, the correlation is assumed to be negligible. For all doping concentrations studied in this work $(0.1 \% \rightarrow 5 \%)$, the FWHM, $\Gamma_{\text {inh }}$, of the profile essentially grows linearly with the concentration $[29,56]$.

In this work each dopant is therefore randomly assigned a frequency based on a Lorentzian line shape whose FWHM grows according to

$$
\Gamma_{\text {inh }}=\Gamma_{0}+\Gamma_{c} c_{\text {total }},
$$

where $\Gamma_{0}=1.8 \mathrm{GHz}$ is a concentration-independent linewidth, $\Gamma_{c}=1800 \mathrm{GHz}[48,56]$, and $c_{\text {total }}$ specifies the total atomic doping concentration between 0 and 1 .

To account for the potentially limited tuning range of the laser used to initialize and control all qubits, any dopants that lie outside this range are now removed. In the present work we assume that the tuning range is either $100 \mathrm{GHz}$ or $1000 \mathrm{GHz}$, and that it is centered around the inhomogeneous absorption profile. The technical challenges of reaching such high laser tunability while still maintaining the coherent and precise control required by the gate operation pulses are discussed further in, e.g., Ref. [27].

A $1000-\mathrm{GHz}$ tuning range is much larger than the maximum linewidth of the inhomogeneous absorption profile studied in this work. For example, doping concentrations of $1 \%$ or $5 \%$ have a linewidth of $19.8 \mathrm{GHz}$ or $91.8 \mathrm{GHz}$, respectively. However, since the absorption profile has a Lorentzian shape [29], many qubits can still be found in the wings of the profile.

Furthermore, for a $1000-\mathrm{GHz}$ tuning range the closeness between the two Eu sites, roughly $150 \mathrm{GHz}$ [57], might complicate the procedure to find qubits when constructing the quantum processor, and it might also increase the effect of ISD. However, if one allows qubits to be formed using either site, the closeness between the two sites might instead be turned into an advantage, as it provides additional ions to be used as qubits. Regardless, in the present work we neglect these issues or benefits and assume that one can isolate $\mathrm{Eu}$ dopants from site 1 , despite the overlap with dopants in site 2 . 


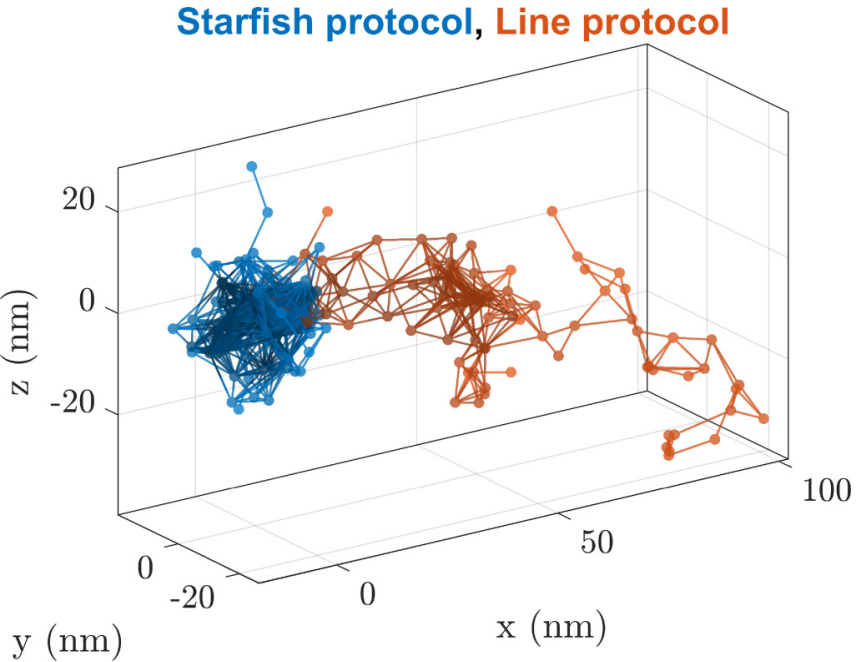

FIG. 2. Shows two examples of the spatial distribution of the qubits in the quantum processor node when constructed using the starfish protocol (blue) and the line protocol (red). The dots represent qubits and the lines represent possible TQ interactions between the connected qubits. In these simulations the doping concentration was $c_{\text {total }}=1 \%$ and the tuning range of the laser was $100 \mathrm{GHz}$.

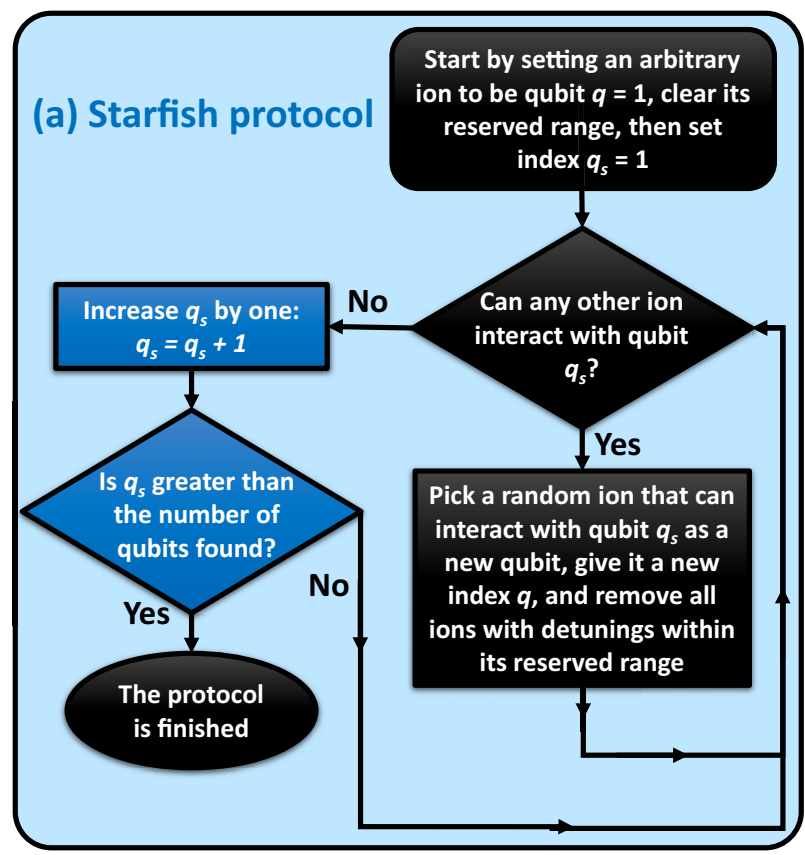

In general, energy transfer processes can occur between any two dopants, but the effect is strongly dependent on the distance between them. In Appendix B we investigate the case for site $1{ }^{153} \mathrm{Eu}: \mathrm{Y}_{2} \mathrm{SiO}_{5}$ and conclude that fluorescence resonant energy transfer effects are negligible.

Finally, each crystal we create is a sphere with a $100-\mathrm{nm}$ radius. Note, however, that a real crystal does not need to be this small in order to achieve the results presented in this work. The radius was picked as a tradeoff between how long the simulations take to run and how wide the qubits are spread out in space. Furthermore, since the creation of the crystal is a stochastic process, we perform 100 different crystal realizations for each protocol or parameter we want to investigate.

\section{Protocols for constructing quantum processor nodes}

In this section we introduce two main protocols to find the ions that are used as qubits in the quantum processor node. We denote these as the starfish and line protocols. In short, the starfish protocol attempts to find new qubits surrounding the qubits found first and therefore results in a dense spatial distribution of the qubits. On the contrary, the line protocol attempts to find new qubits surrounding the qubits found last and hence the qubits form a line of interaction that is more likely to spread out in space. Examples of how the qubits are spatially distributed can be seen in Fig. 2, and flow charts describing the two protocols are given in Fig. 3.

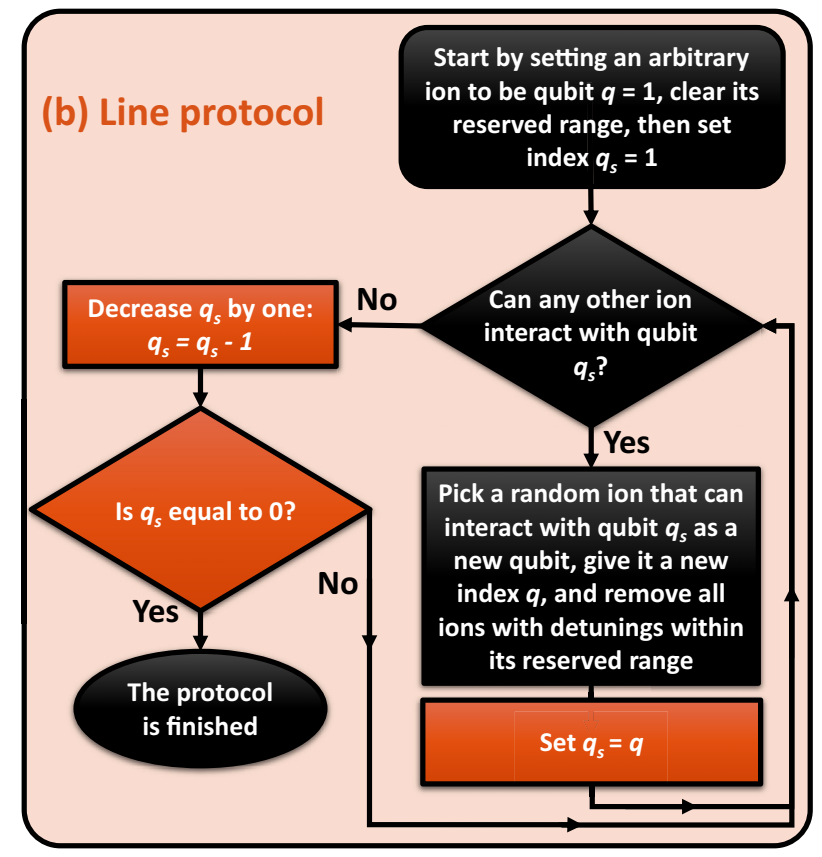

FIG. 3. Shows the procedure to construct quantum processor nodes based on two methods: (a) the starfish protocol (blue) and (b) the line protocol (red), where common steps are shown in black. We give the qubits indices $q=1,2,3 \ldots$ based on the order in which they are found and search for new qubits connected to the qubit with index $q_{s}$. The starfish (line) protocol always attempts to find qubits connected to the first (last) qubit found before continuing the search around later (earlier) qubits. In an experimental setting where the qubits are read out by a dedicated codoped readout ion, the first qubit in the protocol is found by searching the inhomogeneous absorption profile for an ion that can turn off the fluorescence of the readout ion via the dipole-dipole blockade mechanism. Subsequent qubits are also found by searching the inhomogeneous absorption profile, but now looking for ions that can block the excitation of an existing qubit. Since the blockade mechanism only works for sufficiently large dipole-dipole shifts, all interacting qubits are spatially close to each other. Immediately after a qubit is found, it is isolated in frequency space from other nearby nonqubit ions by using optical hole burning techniques to create transmission windows, see Fig. 1(c). For more information about the experimental procedure, see Appendix D. 
In both protocols we find new qubits by randomly choosing one ion that can perform a TQ gate operation with qubit $q_{s}$ that has an error less or equal to $3 \times 10^{-3}$. In the simulations we find suitable candidates by checking if the dipole-dipole shift, $\Delta v$, they cause to qubit $q_{s}$ can be used to perform a TQ gate operation. If the shift is in the range of $0.1 \mathrm{MHz} \leqslant|\Delta v|<$ $7 \mathrm{MHz}$ the interaction gate is used, whereas the blockade gate is used when $|\Delta v| \geqslant 7 \mathrm{MHz}$. However, some shifts are not suitable as they shift the gate operation pulses into resonance with other transitions; for more information see Appendix C.

The ranges listed above are used since they result in TQ gate errors less than or equal to $3 \times 10^{-3}$. However, these ranges are not fixed, and tradeoffs can be made. For example, by decreasing the bandwidth of the pulses used to perform a TQ blockade gate, the minimum requirement of a $7-\mathrm{MHz}$ dipole-dipole shift is decreased. This, however, comes at the cost of longer gate pulses and thus larger errors due to decay and decoherence, which currently limits the fidelity of the TQ gate operation.

The results of the number of qubits and their connections presented in this work are not directly dependent on the fidelities of the SQ and TQ gates. Instead, they depend on the allowed dipole-dipole shifts presented in Appendix C. Thus, as long as the same shifts are allowed, the number of qubits and their connections remain the same, regardless of the SQ and TQ gate fidelities. Furthermore, changes to the lowest range, $-100 \mathrm{kHz} \rightarrow 100 \mathrm{kHz}$, affects the results most since most ions interact weakly due to the $1 /|\boldsymbol{r}|^{3}$ scaling.

In most experimental situations the exact dipole-dipole shifts are not initially known, and how this search can be performed experimentally is therefore still an open question which might even depend on how the state of a qubit is being read out. In Appendix D we present a possible experimental protocol which assumes that codoped readout ions are used to read out the state of qubits via the dipole-dipole blockade mechanism [21,23].

Finally, in Sec. III we also study a modified version of the starfish protocol, namely, the compact starfish. In this protocol one starts by performing the starfish as usual, except one only looks for interactions using the TQ blockade gate. Since this gate requires larger dipole-dipole shifts than the interaction gate, the spatial structure of the compact starfish protocol is even denser than the original version. After no more qubits can be found, the starfish protocol is repeated once more, now searching for more qubits that can interact using either of the TQ gate types, i.e., $q_{s}$ is reset to 1 and the protocol restarts by asking "Can any ion interact with qubit $q_{s}$ ?" in Fig. 3(a), now looking for potential qubits that can perform TQ interaction gates as well.

\section{QUANTUM PROCESSOR NODE PROPERTIES}

In this section we investigate the properties of quantum processor nodes constructed in different ways. We start by examining the connectivity for the line protocol when the doping concentration is $c_{\text {total }}=1 \%$ and the laser tuning range is $100 \mathrm{GHz}$. Figure 4(a) shows the connectivity graph between all qubits for this case. This looks as one could expect based on the spatial distribution seen in Fig. 2 (red line), i.e., each qubit is mainly connected to just a few other qubits in its vicinity, as indicated by the diagonal line in the connectivity graph. It is, however, still possible that qubits clump together and form a slightly more connected subgroup. Furthermore, as it becomes increasingly more difficult to find new qubits connected to the last qubit found, the line protocol dictates that one should try to find qubits connected to earlier qubits, which explains why the qubits with large indices are sometimes connected to earlier qubits and therefore deviate from the main diagonal line.

If the doping concentration increases, the average number of connections increases slightly. The reason for this is that a higher concentration on average leads to shorter distances between qubits, thus increasing the connectivity slightly. The higher concentration also makes it easier to find more qubits. A connectivity graph example for the line protocol where $c_{\text {total }}=5 \%$ can be seen in Fig. 4(b).

Presently, the number of qubits found is limited by the $100-\mathrm{GHz}$ tuning range of the laser. This can be understood, since in the best case each qubit occupies a frequency range of $0.85 \mathrm{GHz}$, thus setting an upper limit on the number of qubits to $\frac{100 \mathrm{GHz}}{0.85 \mathrm{GHz}} \approx 120$. Thus after finding roughly 100 qubits, most optical frequencies within the tuning range are reserved and the probability of finding more ions that are connected to a qubit is low. However, if the tuning range is increased, more qubits can be found. The results of the line protocol with $c_{\text {total }}=5 \%$ and a $1000-\mathrm{GHz}$ tuning range is shown in Fig. 4(c). As can be seen, the overall structure of the connectivity graph remains similar, regardless of tuning range and concentration.

We now turn our investigation toward the starfish protocol. However, this time we begin by examining the case where $c_{\text {total }}=5 \%$ and the laser tuning range is $1000 \mathrm{GHz}$. In the starfish protocol we find all qubits connected to qubit 1 first. In our case, shown in Fig. 4(f), the first qubit can interact with the first roughly 300 qubits. An interaction is only possible if the dipole-dipole shift is at least $\pm 100 \mathrm{kHz}$, see Appendix C, which corresponds to a maximum distance between the qubits in the order of $d=10 \mathrm{~nm}$. For simplicity we can assume that an individual qubit can interact with all qubits within a sphere of radius $d$. Thus the first 300 qubits all lie within the sphere centered at the first qubit. Furthermore, the sphere for any one of those 300 qubits has a large overlap with the sphere centered at the first qubit. Therefore all these qubits form a highly connected subgroup of the processor. This is exactly what we see in Fig. 4(f), where the connectivity graph of these qubits forms a highly filled box.

After all qubits connected to qubit 1 have been found in the starfish protocol, the procedure continues with finding qubits connected to qubit 2 , then qubit 3 , and so on. This creates secondary boxes along the diagonal which are formed due to the same reason as explained above, except the spheres are now centered around qubit 2 , qubit 3 , and so on.

Once more, the number of qubits in the processor becomes limited to roughly 100 if the tuning range of the laser is lowered to $100 \mathrm{GHz}$, as seen in Fig. 4(e). Finally, if the concentration decreases to $1 \%$, the average distance between ions increases and hence fewer qubits are found within the compact spatial distribution of the starfish protocol. It is therefore harder to find more qubits connected to qubit 1 , and the size 
(a)

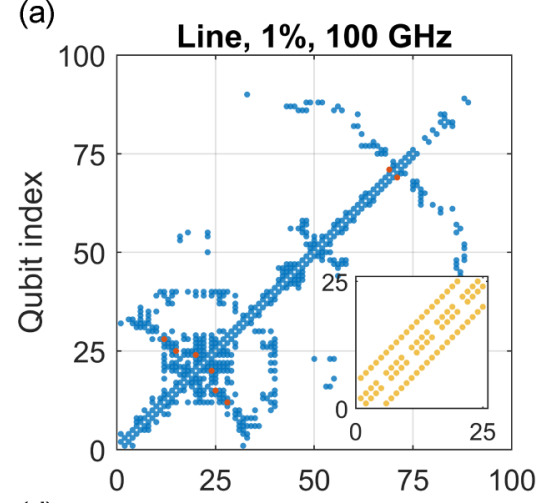$$
\text { (a) }
$$
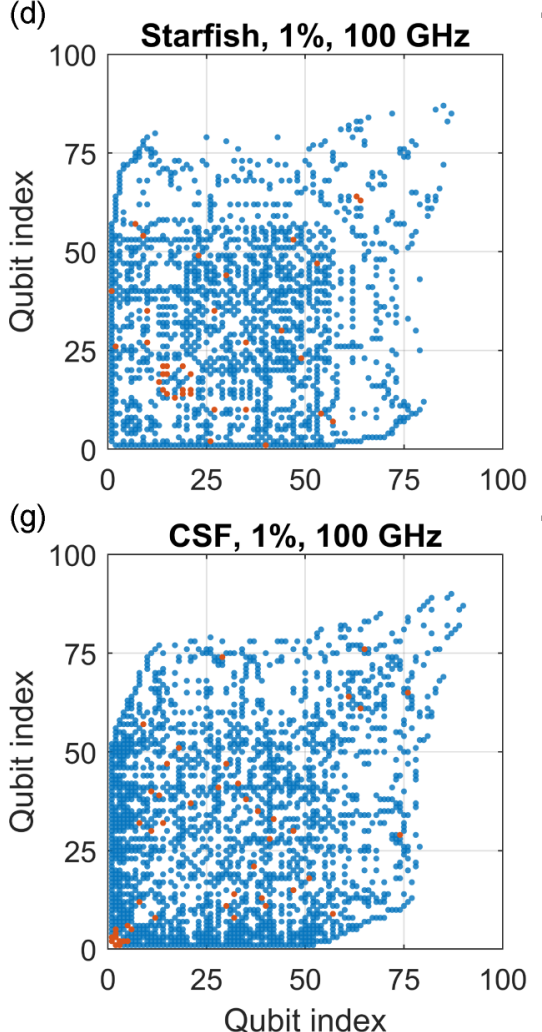

(b)

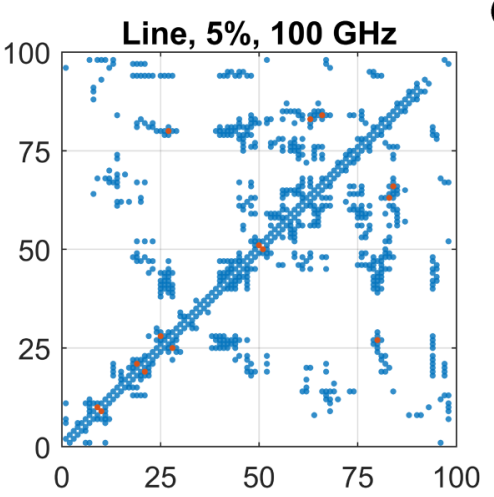

(e)

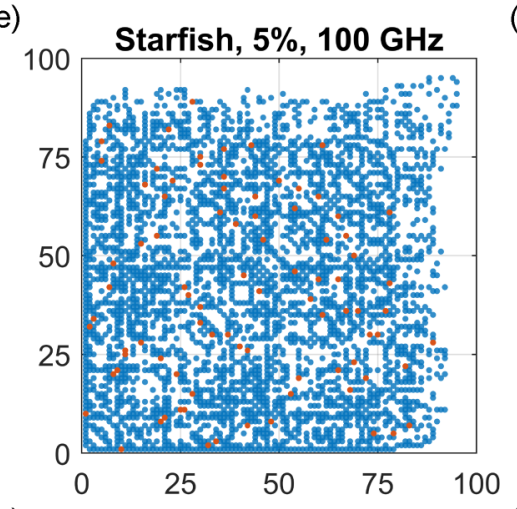

(h)

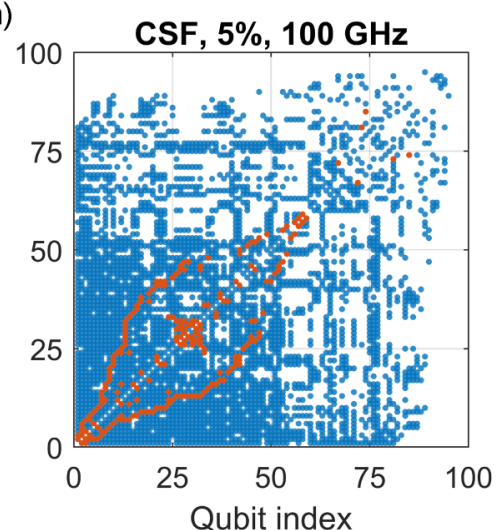

(c)

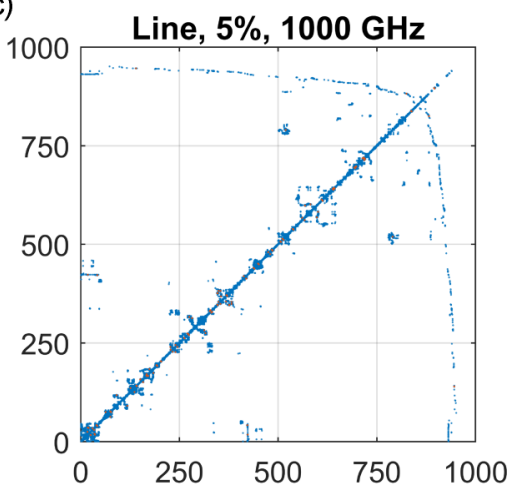

(f)

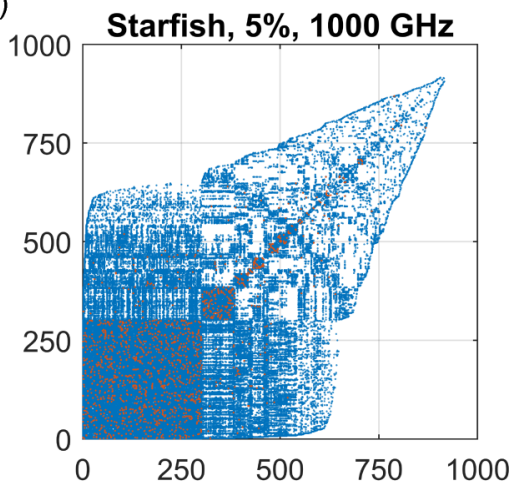

(i)

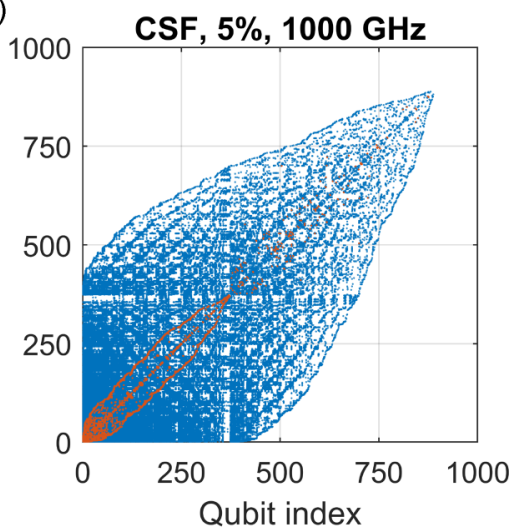

FIG. 4. Shows the connectivity between different qubits, where a blue or red dot at coordinates $(i, j)$ means that the qubits with indices $i$ and $j$ can perform a TQ interaction or blockade gate, respectively. The titles for each graph explain which protocol (CSF $=$ compact starfish), doping concentration $c_{\text {total }}$, and laser tuning range were used in the construction of the processor node. These graphs show the result from one crystal realization each but still represent typical cases for how the connectivity is distributed depending on the parameters. In order to easier compare to the connectivity of other systems, we in the inset of graph (a) show the connectivity graph for the case of qubits arranged in a square grid $(5 \times 5$ grid for a total of 25 qubits $)$ where each qubit is connected to its immediate neighbors to the left, right, up, and down. This results in four lines where the two central lines are cut at regular intervals since the qubits at the edges (corners) of the grid are missing one (two) neighbor(s). For a system where all qubits can directly interact with all other qubits, the connectivity graph would be completely filled. $(a-c)$ The main diagonal line arises since the line protocol always attempts to find new qubits connected to the latest qubit found, see Fig. 3(b). (d-f) The visual square-shaped patterns arise when many qubits form a highly connected subgroup. This occurs since the starfish protocol attempts to find as many qubits as possible connected to one qubit, see Fig. 3(a), and all those qubits are therefore spatially close to each other. For more detailed explanations, see the main text.

of the connectivity box thus shrinks, as seen when comparing the results of Figs. 4(d) and 4(e). Figures 4(g)-4(i) show the results of the compact starfish protocol, which is discussed more at the end of this section.

The connectivities of the starfish and compact starfish protocols are much greater than the line protocol. There is, however, a potential benefit in using the line protocol. The limiting factor on the number of qubits in the processor node is directly related to the number of available frequency channels, i.e., the ratio between the tuning range of the laser and the size of the frequency interval each qubit reserves. However, if the laser used to control the qubits has a small enough focus size compared to the spatial extent of the qubits, one can translate the laser focus so that the light no longer interacts with some of the qubits. The reserved frequency range of those qubits can therefore be reused, since qubits only interact with 

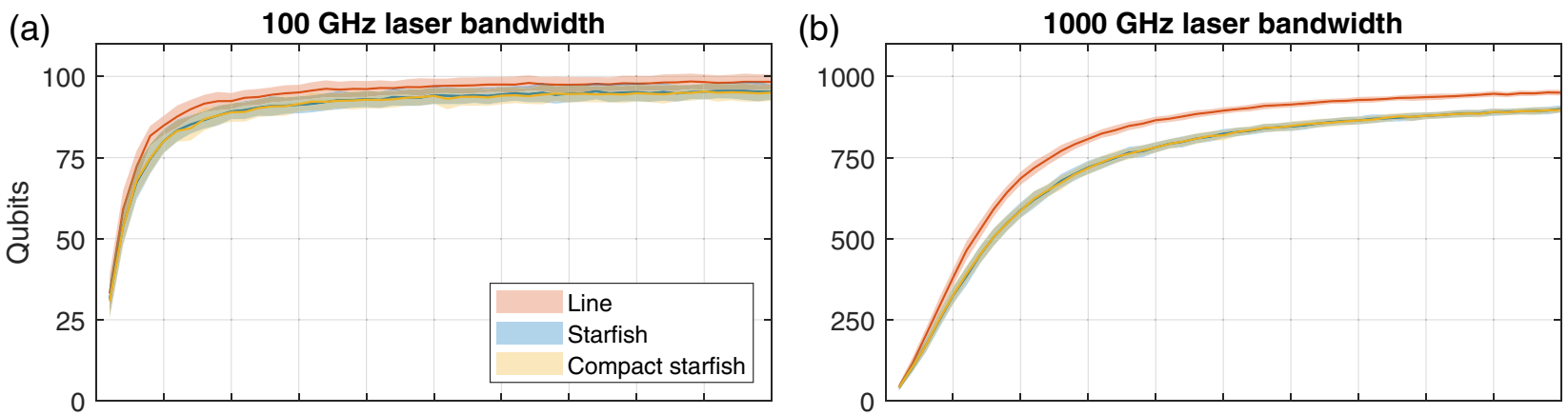

(c)

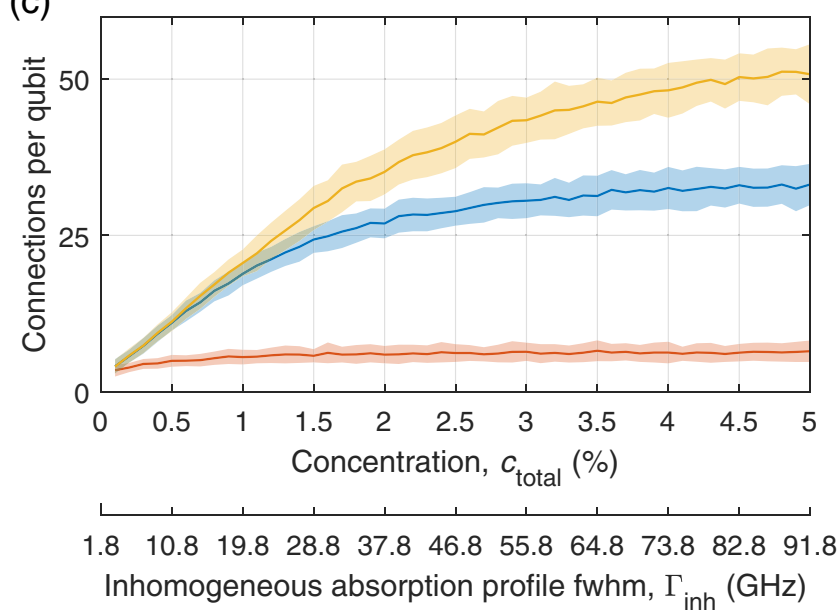

(d)

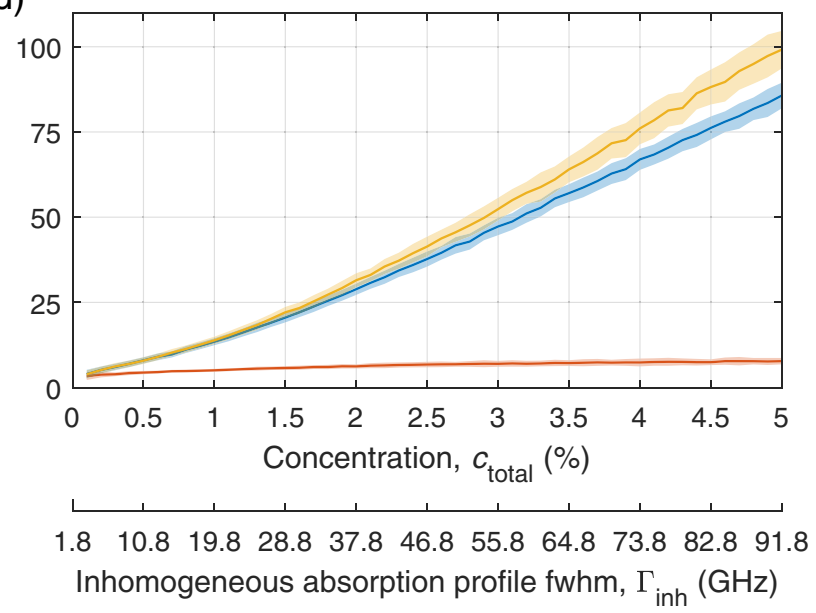

FIG. 5. Shows the $(a, b)$ average number of qubits and (c, d) average number of connections per qubits in the processor node as a function of the total doping concentration, $c_{\text {total }}$, and the corresponding FWHM of the inhomogeneous absorption profile, $\Gamma_{\text {inh }}$, which is calculated from Eq. (2). Each data set is constructed from investigating 100 different crystal realizations, where the solid lines show the average result and the transparent regions show one standard deviation. In (a) and (c) the laser tuning range is limited to $100 \mathrm{GHz}$, whereas the range is $1000 \mathrm{GHz}$ in (b) and (d). In all cases the tuning range is centered on the inhomogeneous absorption profile. The red (blue/yellow) data uses the line (starfish/compact starfish) protocol to construct the processor node.

the laser if both the correct frequency and the correct spatial location of the laser focus is used. This opens up possibilities for future protocols which scale beyond the constrains of a single processor node, e.g., by using thin-film crystals and a translatable laser focus.

Finally, a common feature to all connectivity graphs is that the number of TQ interaction gates (blue dots) heavily outnumbers the number of TQ blockade gates (red dots). This occurs since TQ interaction gates work for smaller dipoledipole shifts and thus larger distances compared to the TQ blockade gates.

Figure 4 examines how the connectivity of processors might look in a few different cases. Now we instead investigate the number of qubits and connections per qubit in the processor when averaging the data of 100 different crystal realizations. The results for all protocols is shown in Fig. 5 as a function of the doping concentration.

As can be seen in Fig. 5(a), the line protocol (red) results on average in slightly more qubits compared to the starfish protocol (blue). Furthermore, as the doping concentration increases from $c_{\text {total }}=0.1 \%$ to $1 \%$, the number of qubits in the processor grows from roughly 30 to 90 . However, as the concentration continues to grow the number of qubits remains fairly constant at around 100 . The reason for this saturation is the limited tuning range of $100 \mathrm{GHz}$, as explained previously.
If the tuning range is increased to $1000 \mathrm{GHz}$ up to ten times more qubits can be found, as is seen in Fig. 5(b).

Since there are more ions with resonance frequencies close to the center of the inhomogeneous absorption profile, see Fig. 1(d), it is more likely that those frequency channels are reserved first. However, each time we select another qubit we at least reserve a frequency range of $0.85 \mathrm{GHz}$ to that qubit. Thus, to find more qubits we must find ions in the outskirts of the inhomogeneous absorption profile. The initial increase in the number of qubits when the concentration grows from a low value can therefore be explained by an increased probability of finding ions with large detunings from the inhomogeneous absorption profile that also lie sufficiently close in space to an already existing qubit in the processor.

We now continue our investigation by examining the average number of connections each qubit has, i.e., how many other qubits can a qubit interact with using the TQ gate operations. This is shown in Figs. 5(c) and 5(d) for laser tuning ranges of $100 \mathrm{GHz}$ and $1000 \mathrm{GHz}$, respectively. Here the difference between the line and starfish protocols is more evident, with the starfish protocol having many more connections per qubit since the ions form a more compact spatial formation, as seen in Fig. 2. Furthermore, even though the number of qubits in the processor saturates at a fairly low concentration, the average number of connections in the starfish 
protocol continues to grow for much longer. As explained previously, this is also visualized by the increased size of the box in the connectivity graph as shown in Fig. 4(e).

When increasing the doping concentration two effects occur simultaneously: the total number of dopants increases, and the inhomogeneous absorption profile is broadened as determined by Eq. (2). In Appendix E we investigate how these effects individually affect the number of qubits and the connectivity of the processor node. The conclusions are that increasing the width of the inhomogeneous absorption profile can result in more qubits and more connections, but mainly if the width was originally much less than the tuning range of the laser. The width can, e.g., be increased by codoping the crystal with another rare-earth ion [58]. Furthermore, increasing the number of dopants without broadening the inhomogeneous absorption profile always leads to more qubits and connections. However, neither effect impacts the number of qubits if it is already saturated by the limited tuning range of the laser.

Finally, the line and starfish protocols presented in this work can be modified or combined in order to partly obtain control over how the final connectivity graph should look. One example of this is the compact starfish protocol, which at first only allows interactions via TQ blockade gates as explained in Sec. IIC. Thanks to its denser spatial structure even when compared to the original starfish protocol, this modified protocol achieves more connections per qubit on average, as can be seen in Figs. 5(c) and 5(d). For completeness we also provide a few examples of the connectivity graphs for this protocol, see Figs. 4(g)-4(i).

\section{CONCLUSION}

In this work we have presented two main protocols, as well as a modified protocol, to construct quantum processor nodes using randomly doped rare-earth-ion crystals. The compact starfish protocol generates the densest spatial distribution of the qubits and thus has more connections per qubit on average. For a laser with a tuning range of $100 \mathrm{GHz}$, this protocol achieves almost 100 qubits with roughly 50 connections per qubit. Furthermore, if the tuning range is increased to $1000 \mathrm{GHz}$, these properties are increased to almost 1000 qubits with roughly 100 connections per qubit on average.

We also conclude that increasing the width of the inhomogeneous absorption profile can be beneficial as long as the width is less than the tuning range of the laser. Furthermore, increasing the doping concentration up to at least $5 \%$ was always beneficial as it increased the number of connections between qubits.

These results assume gate operation parameters that have numerically been shown to have low gate errors $[28,33]$. Thus the processor nodes with the properties listed above should be ready for error correction. Our vision is then that several nodes can be connected to each other in a multinode architecture in order to further scale up the number of qubits, e.g., via optical interfaces and communication by flying qubits in the form of light $[27,59]$.

Finally, the line protocol has fewer connections on average, but its qubits are spread out more in space. This opens up other possibilities for future scaling beyond the limits of a single processor node. For example, a translatable laser that is focused onto a small spot on a thin film could allow for the reuse of qubit frequency channels, as each qubit is only interacting with the laser light if the correct frequency and the correct spatial position of the laser focus is used.

\section{ACKNOWLEDGMENTS}

This research was supported by the Swedish Research Council (Grants No. 2016-05121, No. 2015-03989, No. 201604375, and No. 2019-04949), the Knut and Alice Wallenberg Foundation (KAW 2016.0081), the Wallenberg Center for Quantum Technology (WACQT) funded by the Knut and Alice Wallenberg Foundation (KAW 2017.0449), and has received funding from the European Union's Horizon 2020 research and innovation programme under Grant Agreement No. 820391 (SQUARE).

\section{APPENDIX A: CALCULATING THE RESERVED FREQUENCIES FOR A QUBIT}

In this section we obtain which frequencies each qubit must reserve so that transmission windows can be created for all qubits. Note that this requirement is only necessary if instantaneous spectral diffusion (ISD) is deemed to be a problem and therefore should be minimized, which we assume for the present work. The procedure to calculate these reserved frequencies is shown in Fig. 6. The conclusion is that a single qubit, shown in blue, reserves the frequency intervals shown in graph (c). In total these intervals cover roughly $1.7 \mathrm{GHz}$. However, the graph only shows what frequencies are forbidden for the $|0\rangle \rightarrow|e\rangle$ transition of another qubit. Therefore the reserved frequencies of different qubits may partly overlap. In the best case roughly half of the frequency intervals can overlap, which corresponds to a qubit reserving $1.7 / 2 \mathrm{GHz}=0.85 \mathrm{GHz}$, which cannot be used by another qubit.

\section{APPENDIX B: ENERGY TRANSFER BETWEEN EU DOPANTS IN $\mathrm{Y}_{2} \mathrm{SiO}_{5}$}

In general, the fluorescence resonant energy transfer (FRET) rate for a dipole-dipole interaction between two dopants is given by [60-62]

$$
A_{\mathrm{ET}}=\frac{C_{d d}}{|\boldsymbol{r}|^{6}},
$$

where $|\boldsymbol{r}|$ is the distance between the dopants, and $C_{d d}$ is the transfer microparameter for a dipole-dipole interaction and is calculated using [63]

$$
C_{d d}=\frac{3 c}{8 \pi^{4} n^{2}} \int \sigma_{\mathrm{Em}, \mathrm{D}}(\lambda) \sigma_{\mathrm{Abs}, \mathrm{A}}(\lambda) d \lambda,
$$

where $\sigma_{\mathrm{Em}, \mathrm{D}}$ and $\sigma_{\mathrm{Abs}, \mathrm{A}}$ are the donor emission and acceptor absorption cross sections, respectively, $c$ is the speed of light in vacuum, and $n$ is the index of refraction of the host, which is 1.78 for $\mathrm{Y}_{2} \mathrm{SiO}_{5}$ [64].

Averaged absorption cross sections for the ${ }^{7} F_{0} \rightarrow{ }^{5} D_{0}$ transition for both Eu sites were obtained by measuring the polarized absorption spectra along all three principal axes, where some parts of the polarization-averaged spectra are 


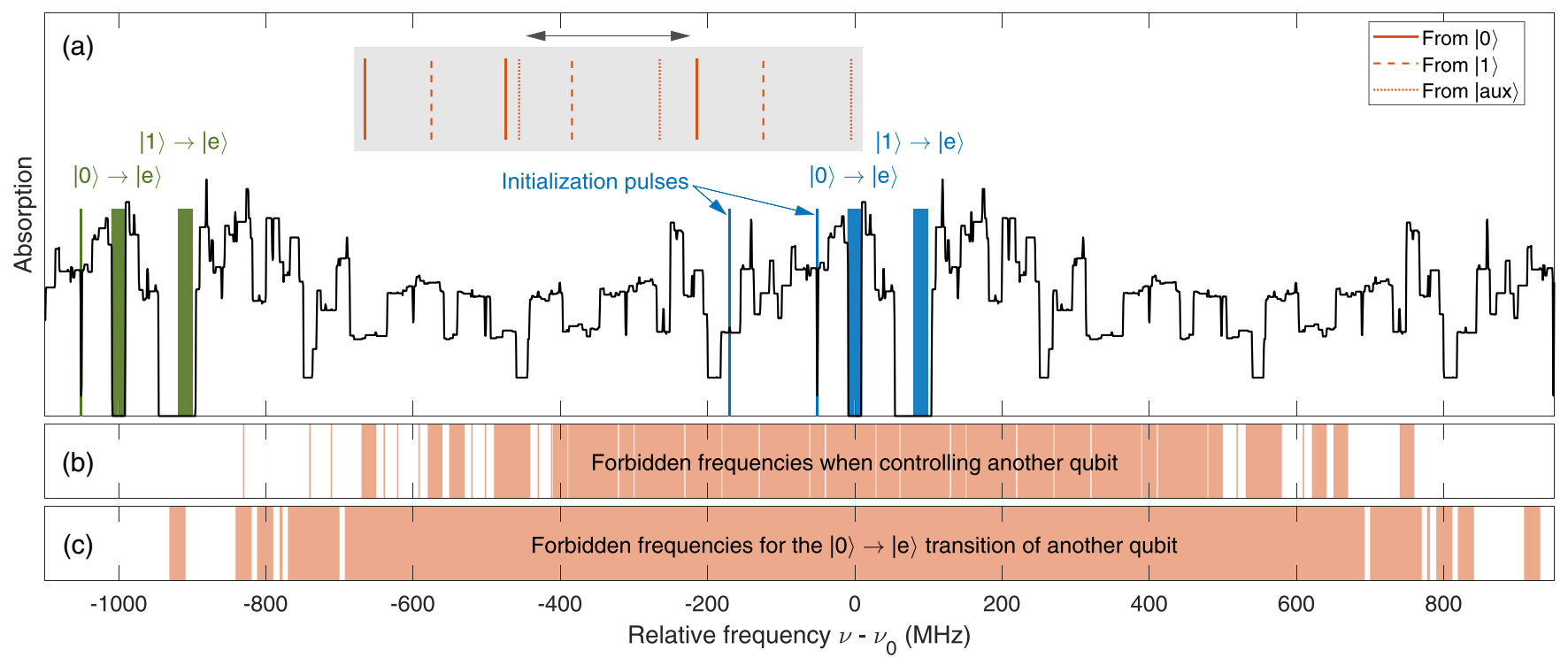

FIG. 6. (a) Shows the absorption spectrum as a function of the relative frequency $v-v_{0}$, where $v_{0}$ is the $|0\rangle \rightarrow|e\rangle$ transition frequency of qubit 0 . The blue regions indicate where we might send in light in order to control or reinitialize qubit 0 . The wider regions are $20 \mathrm{MHz}$ broad and cover the frequency bandwidth of the gate operation pulses, whereas the narrower regions (indicated by the blue arrows) are only $2 \mathrm{MHz}$ broad and are only used to initialize the qubit from $|\mathrm{aux}\rangle \rightarrow|3 / 2 \mathrm{e}\rangle \rightarrow|1\rangle$. In order to minimize instantaneous spectral diffusion (ISD), the pulses controlling a second qubit, shown in green, should not contain frequencies which could optically pump nonqubit ions back into any of the frequencies used to control the blue qubit. We now calculate which those frequencies are. In the gray box we show the nine transition frequencies of a nonqubit ion (red), where solid/dashed/dotted lines show transitions from the $|0\rangle /|1\rangle / \mid$ aux $\rangle$ ground state, respectively [the energy-level structure used to calculate the transition frequencies is shown in Fig. 1(a)]. We translate the gray box in frequency, and anytime a transition frequency overlaps with one of the blue regions we mark the frequencies of the red transitions from the two other ground states as forbidden. For example, the highest frequency transition in the gray box (rightmost dotted red line), which indicates a transition from the $|\mathrm{aux}\rangle$ state, overlaps with a blue region. Therefore we are not allowed to use any frequency where a transition from either $|0\rangle$ (solid red lines) or $|1\rangle$ (dashed red lines) exists, since that might optically pump the nonqubit ion into $|\mathrm{aux}\rangle$, from where it may cause significant ISD errors when an operation is performed on the blue qubit. (b) Frequencies that are not allowed when controlling another qubit, as obtained by the method described above. Hence the green regions, which are the frequencies used to control another qubit, are not allowed to overlap with these frequencies. Since it is known which four frequency intervals we must use to control the green qubit, it is possible to calculate which frequencies are forbidden for the $|0\rangle \rightarrow|e\rangle$ transition of the green qubit. These frequencies are shown in graph (c). In conclusion, the $|0\rangle \rightarrow|e\rangle$ transition of another qubit cannot have a frequency that overlaps with the spectrum shown in graph (c).

published in Ref. [62]. The data was obtained at $10 \mathrm{~K}$ using a Varian Cary 6000i spectrophotometer with a $0.1 \mathrm{~nm}$ resolution.

The ${ }^{5} D_{0} \rightarrow{ }^{7} F_{0}$ emission cross sections were estimated based on the data published in [65]. The data was recorded using an Oriel spectrometer (MS125) with an Andor CCD camera as detector, the sample was kept at $4 \mathrm{~K}$, and the excitation was performed at $370 \mathrm{~nm}$. The spectral resolution is estimated to be around $0.5 \mathrm{~nm}$. Both the emission and absorption measurements were recorded in a $0.1 \% \mathrm{Eu}: \mathrm{Y}_{2} \mathrm{SiO}_{5}$ crystal.

From these results the $C_{d d}$ parameter could be calculated: $C_{d d} \approx 0.43 \mathrm{~nm}^{6} / \mathrm{s}$ for site 1 (and $\approx 0.24 \mathrm{~nm}^{6} / \mathrm{s}$ for site 2 , but this value is not relevant for this work). The value provides a rough order of magnitude, and even in the worst case we estimate that the value does not grow by more than a factor of 6 .

Finally, energy transfer effects are only significant if $A_{\mathrm{ET}}$ is of similar size compared to the radiative decay set by $A_{\text {rad }}=$ $1 / T_{1}$, where $T_{1}=1.9 \mathrm{~ms}$ for the optical transition [57], i.e., $A_{\mathrm{ET}} \approx A_{\text {rad. }}$. This occurs when the distance between the two dopants is $|\boldsymbol{r}| \approx\left(C_{d d} T_{1}\right)^{1 / 6} \approx 0.3 \mathrm{~nm}$. The shortest distance between two dopants in $\mathrm{Y}_{2} \mathrm{SiO}_{5}$ is roughly $0.35 \mathrm{~nm}$, and since most dopants are much farther apart than this, energy transfer effects are assumed to be negligible. The same reasoning applies to energy transfer by exchange interaction, which is only relevant at very short ion-ion distances (typically a few Ångström) [66]. The expected low impact of energy transfer mechanisms in our scheme is further supported by the long $\mathrm{Eu}$ excited-state lifetimes measured in stoichiometric Eu crystals despite the short Eu-Eu distances [67,68], i.e., quenching is absent.

\section{APPENDIX C: UNUSABLE DIPOLE-DIPOLE SHIFTS FOR TQ GATE OPERATIONS}

When performing TQ blockade gate operations, if the dipole-dipole shift, $\Delta v$, is such that it moves another transition from either $|0\rangle$ or $|1\rangle$ into resonance with one of the gate operation pulses, the TQ gate does not work; see Ref. [28] for more details. Furthermore, when performing TQ interaction gate operations, if $|\Delta v|<100 \mathrm{kHz}$ the operation takes too long and thus the error is too high. Therefore the simulations in this work do not look for interactions between qubits if the dipole-dipole shift is in one of the ranges shown in Fig. 7. For 


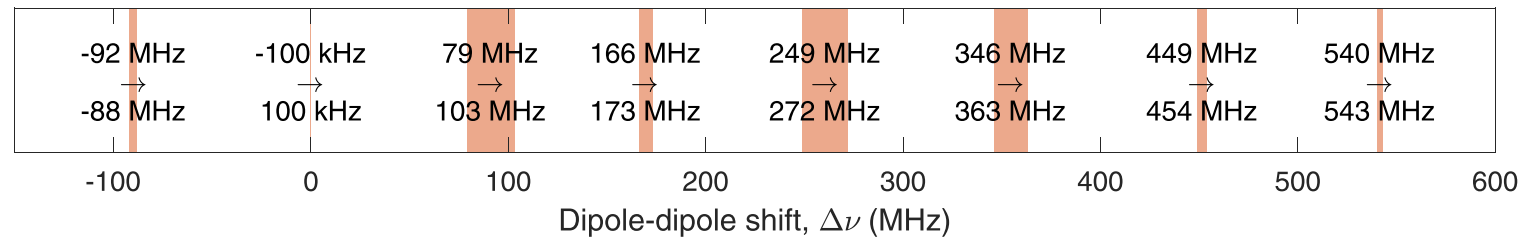

FIG. 7. In order for the TQ gate errors of the blockade and interaction gates with gate parameters found in Ref. [28] to be lower than or equal to $3 \times 10^{-3}$, the dipole-dipole shift, $\Delta v$, between two interacting qubits cannot lie in any of the intervals listed above.

any other dipole-dipole shift the TQ gate error is less than or equal to $3 \times 10^{-3}$.

\section{APPENDIX D: AN EXPERIMENTAL PROTOCOL FOR CONSTRUCTING QUANTUM PROCESSOR NODES}

In this section we present a protocol for constructing a quantum processor node in an experimental setting. The method assumes that codoped readout ions are used to read out the state of qubits via the dipole-dipole blockade mechanism [21,23].

First, a readout ion with strong fluorescence is identified. Then one should find a qubit ion that can turn this fluorescence off via the dipole-dipole blockade mechanism. This is done by exciting various frequency intervals in the inhomogeneous absorption profile of the qubit ions and seeing if the readout fluorescence disappears. A suitable frequency width of the intervals might be between $1 \mathrm{MHz}$ and $10 \mathrm{MHz}$, depending on how likely it is to find a qubit ion that can block the readout. If the fluorescence disappears, it means that at least one qubit ion with a transition within the frequency interval has a sufficiently strong interaction with the readout ion, i.e., it is sufficiently close in space.

The next step is to determine which transition the potential qubit ion was excited on. Optical pumping techniques [19, 30-32] can be used to force the ion into a specific frequency interval if an initial guess of its transition frequency is correct. By then repeating the excitation within that frequency interval one can determine if the assumed transition was correct or not depending on if the fluorescence disappears or not. If not, one repeats the procedure by assuming that the qubit ion was originally excited on one of the other optical transitions.

After finding the correct transition, one should narrow the uncertainty of its transition frequency by exciting eversmaller frequency intervals. Following this, optical pumping techniques can be used to create transmission windows surrounding the two qubit transitions to isolate them in frequency space. Then SQ gate operation can be benchmarked to optimize the optical frequencies and Rabi frequencies of the two qubit transitions. Both the search procedure and the narrowing of uncertainty in the transition frequencies might be sped up by using binary search methods.

The protocol proceeds by finding a second qubit that can interact with the first qubit. The method is similar to that described above, except we do not search the frequency intervals that lie within the reserved intervals for the first qubit as described in Appendix A since this would destroy the transmission windows of the first qubit, and we now look for a new qubit ion that can block a NOT operation on the first qubit. In more detail, we initialize the first qubit in state $|1\rangle$, excite a frequency interval as above, attempt to perform a NOT operation on the first qubit, and lastly, after the ions in the excited frequency interval have decayed to the ground state, we again attempt to excite the first qubit from state $|1\rangle$ and see if the readout fluorescence is turned off or not. If it turns off, we know that at least one ion within the frequency interval interacts strongly enough with the first qubit to prevent the NOT operation, since if the NOT operation works the first qubit moves to state $|0\rangle$ and is therefore never excited, thus the readout fluorescence is never turned off. The reason we wait for the ions in the frequency interval to decay from the excited state is that they otherwise may be able to directly block the readout ion, which is not what we are searching for in this protocol. Similarly as above, we then narrow the uncertainty in the optical frequency of the second qubit before we benchmark its properties, including TQ gate operations with the first qubit.

In order for this protocol to find qubits that interact even when the dipole-dipole shift between two qubits is small, $0.1 \mathrm{MHz} \leqslant|\Delta v|<7 \mathrm{MHz}$, the NOT operations performed above must have narrower bandwidth and thus longer gate durations than the normal SQ gate operations. This is, however, not a problem, since the increased error on the NOT operation only comes in when we are searching for new qubits and therefore does not affect the performance of running algorithms on the processor node once it has been constructed.

The procedure described above can be generalized to find more qubits until no more frequency intervals are available or until no more qubits can be found. The final step of constructing the quantum processor node is to benchmark all SQ and TQ gates, including TQ gates between qubits that never interacted during the search protocol, and determine which qubits can be directly read out by the readout ion. Finally, note that even if the construction of the quantum processor node would require many pulses and take a long time, the search only has to be done once.

\section{APPENDIX E: INVESTIGATING THE IMPACT OF INCREASING THE DOPING CONCENTRATION}

This section examines why the number of qubits and their connectivity changes when the doping concentration changes. Note that two effects occur simultaneously when the concentration is increased: the total number of dopants increases, and the inhomogeneous absorption profile is broadened as determined by Eq. (2). To investigate these effects we perform new simulations using the starfish protocol. First, the doping concentration is kept fixed at $c_{\text {total }}=1 \%$, but the inhomogeneous absorption profile is still being broadened. Experimentally, this can be achieved by, e.g., codoping the crystal with another rare-earth species [58]. Second, the concentration changes but 

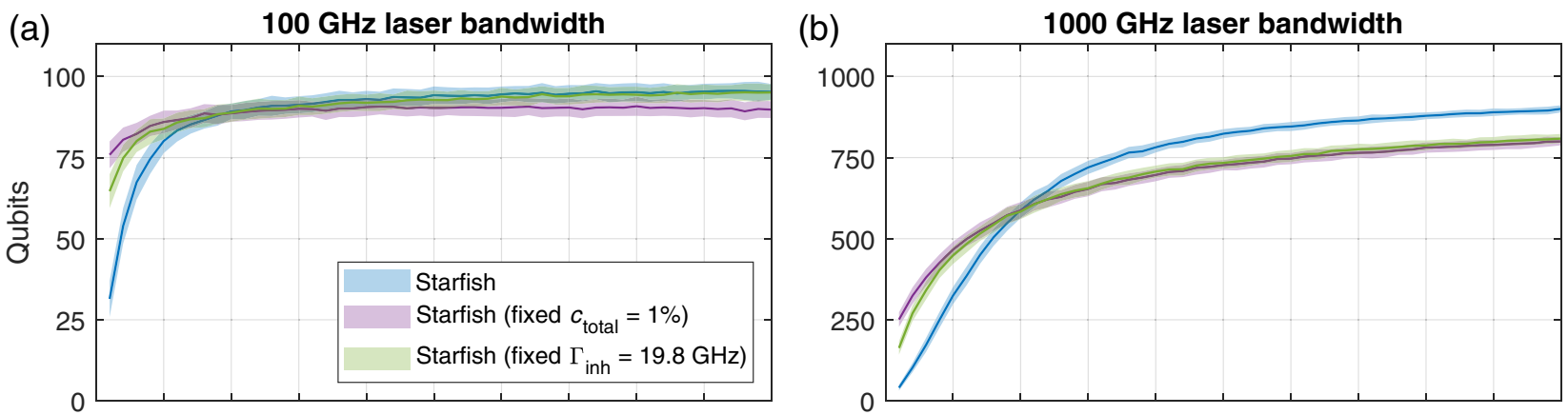

(c)

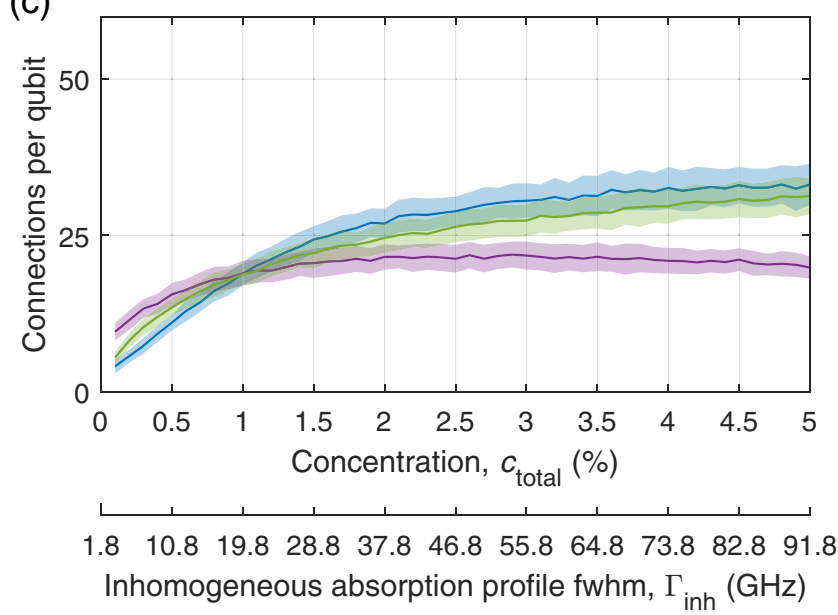

(d)

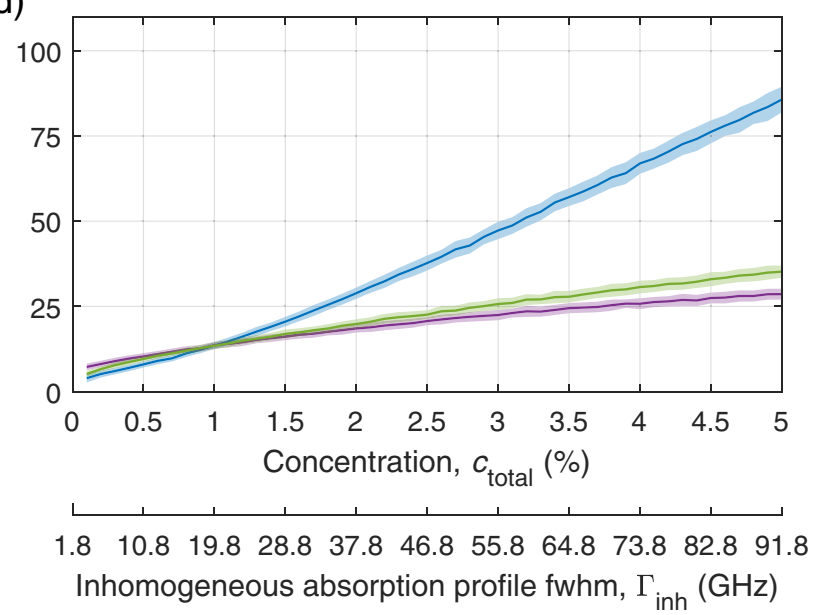

FIG. 8. Shows similar graphs as Fig. 5, but we now only study the starfish protocol. The purple data keeps the doping concentration fixed at $c_{\text {total }}=1 \%$ and only varies the FWHM of the inhomogeneous absorption profile. For this data one should thus only use the $\Gamma_{\text {inh }}$ horizontal axes. Note that experimentally it might be difficult to achieve the purple data for $\Gamma_{\text {inh }}<19.8 \mathrm{GHz}$, since this is the minimum FWHM as set by Eq. (2) for a doping concentration of $c_{\text {total }}=1 \%$. Lastly, the green data keeps the inhomogeneous absorption width fixed at $19.8 \mathrm{GHz}$ and only varies the concentration, and hence only the concentration axes should be used for this data. Note that it might experimentally be difficult to achieve the green data for concentrations $c_{\text {total }}>1 \%$ since increasing the concentration normally increases the inhomogeneous broadening according to Eq. (2).

the FWHM of the inhomogeneous absorption profile, $\Gamma_{\text {inh }}$, is kept fixed at $19.8 \mathrm{GHz}$, which is the width the profile has for a $1 \%$ doping concentration in Eq. (2). This second case is difficult to realize experimentally, since increasing the doping concentration normally increases the inhomogeneous broadening. Therefore this case should primarily be used as a tool to investigate the effect of purely increasing the number of dopants in the crystal. The simulation results are shown in Fig. 8.

We start by examining the fixed doping concentration case (purple data) for the $100-\mathrm{GHz}$ laser tuning range, see Figs. 8(a) and 8(c). Here the number of qubits in the processor has already saturated for the fixed $1 \%$ doping concentration, and neither the number of qubits nor the number of connections increase as the inhomogeneous width is increased above 19.8 GHz. In other words, the effect of increasing the inhomogeneous width is negligible for a $1 \%$ doping concentration and a $100-\mathrm{GHz}$ tuning range. However, for the $1000-\mathrm{GHz}$ tuning range case, both the number of qubits and connections increase as the inhomogeneous absorption profile is broadened, as is seen in Figs. 8(b) and 8(d).

For the fixed inhomogeneous width (green data), the number of qubits exhibits similar behavior as the fixed con- centration case. However, even in the $100-\mathrm{GHz}$ case the number of connections now continues to grow as the concentration is increased beyond $1 \%$.

In order to explain these effects, it is useful to examine how many qubits are connected to qubit 1 . When the concentration is fixed and only $\Gamma_{\text {inh }}$ increases, the number of ions, and thus also the number of qubits, in the center of the inhomogeneous absorption profile decreases, whereas the numbers increase in the outskirts of the profile. For the 100-GHz tuning range case these two effects compensate each other and the total number of qubits connected to qubit 1 remains fairly constant even when $\Gamma_{\text {inh }}$ changes. Therefore the size of the connectivity box shown in Fig. 4(d) remains roughly the same size, which explains why the number of connections per qubit does not increase when $\Gamma_{\text {inh }}$ increases in the $100-\mathrm{GHz}$ tuning range case.

When we instead have a fixed width and only the doping concentration increases, the number of ions at all frequencies increases. Therefore the probability of finding new qubits at any transition frequency increases. Hence the number of qubits connected to qubit 1 increases, the connectivity box increases, and the average number of connections per qubit increases. 
In the 1000-GHz tuning range cases the same explanations are valid, except that now when the concentration is fixed and only $\Gamma_{\text {inh }}$ increases the additional number of qubits found in the outskirts of the inhomogeneous absorption profile out- weighs the decrease in the number of qubits in the center of the profile. This is why the number of qubits and the number of connections in the $1000-\mathrm{GHz}$ fixed concentration case continues to grow.
[1] M. Nilsson and S. Kröll, Solid state quantum memory using complete absorption and re-emission of photons by tailored and externally controlled inhomogeneous absorption profiles, Opt. Commun. 247, 393 (2005).

[2] B. Kraus, W. Tittel, N. Gisin, M. Nilsson, S. Kröll, and J. I. Cirac, Quantum memory for nonstationary light fields based on controlled reversible inhomogeneous broadening, Phys. Rev. A 73, 020302(R) (2006).

[3] G. Hetet, J. J. Longdell, A. L. Alexander, P. K. Lam, and M. J. Sellars, Electro-Optic Quantum Memory for Light Using TwoLevel Atoms, Phys. Rev. Lett. 100, 023601 (2008).

[4] H. de Riedmatten, M. Afzelius, M. U. Staudt, C. Simon, and N. Gisin, A solid-state light-matter interface at the single-photon level, Nature (London) 456, 773 (2008).

[5] M. Afzelius, I. Usmani, A. Amari, B. Lauritzen, A. Walther, C. Simon, N. Sangouard, J. Minar, H. de Riedmatten, N. Gisin, and S. Kröll, Demonstration of Atomic Frequency Comb Memory for Light with Spin-Wave Storage, Phys. Rev. Lett. 104, 040503 (2010).

[6] M. P. Hedges, J. J. Longdell, Y. Li, and M. J. Sellars, Efficient quantum memory for light, Nature (London) 465, 1052 (2010).

[7] S. E. Beavan, M. P. Hedges, and M. J. Sellars, Demonstration of Photon-Echo Rephasing of Spontaneous Emission, Phys. Rev. Lett. 109, 093603 (2012).

[8] M. Sabooni, Q. Li, S. Kröll, and L. Rippe, Efficient Quantum Memory Using a Weakly Absorbing Sample, Phys. Rev. Lett. 110, 133604 (2013).

[9] J. Dajczgewand, J. L. Le Gouet, A. Louchet-Chauvet, and T. Chaneliere, Large efficiency at telecom wavelength for optical quantum memories, Opt. Lett. 39, 2711 (2014).

[10] M. Gündoğan, P. M. Ledingham, K. Kutluer, M. Mazzera, and H. de Riedmatten, Solid State Spin-Wave Quantum Memory for Time-Bin Qubits, Phys. Rev. Lett. 114, 230501 (2015).

[11] P. Jobez, C. Laplane, N. Timoney, N. Gisin, A. Ferrier, P. Goldner, and M. Afzelius, Coherent Spin Control at the Quantum Level in an Ensemble-Based Optical Memory, Phys. Rev. Lett. 114, 230502 (2015).

[12] D. Schraft, M. Hain, N. Lorenz, and T. Halfmann, Stopped Light at High Storage Efficiency in a $\operatorname{Pr}^{3+}: \mathrm{Y}_{2} \mathrm{SiO}_{5}$ Crystal, Phys. Rev. Lett. 116, 073602 (2016).

[13] C. O'Brien, N. Lauk, S. Blum, G. Morigi, and M. Fleischhauer, Interfacing Superconducting Qubits and Telecom Photons via a Rare-Earth-Doped Crystal, Phys. Rev. Lett. 113, 063603 (2014).

[14] L. A. Williamson, Y. H. Chen, and J. J. Longdell, MagnetoOptic Modulator with Unit Quantum Efficiency, Phys. Rev. Lett. 113, 203601 (2014).

[15] X. Fernandez-Gonzalvo, S. P. Horvath, Y. H. Chen, and J. J. Longdell, Cavity-enhanced raman heterodyne spectroscopy in $\mathrm{Er}^{3+}: \mathrm{Y}_{2} \mathrm{SiO}_{5}$ for microwave to optical signal conversion, Phys. Rev. A 100, 033807 (2019).

[16] G. J. Pryde, M. J. Sellars, and N. B. Manson, Solid State Coherent Transient Measurements Using Hard Optical Pulses, Phys. Rev. Lett. 84, 1152 (2000).
[17] K. Ichimura, A simple frequency-domain quantum computer with ions in a crystal coupled to a cavity mode, Opt. Commun. 196, 119 (2001).

[18] N. Ohlsson, R. K. Mohan, and S. Kröll, Quantum computer hardware based on rare-earth-ion-doped inorganic crystals, Opt. Commun. 201, 71 (2002)

[19] M. Nilsson, L. Rippe, N. Ohlsson, T. Christiansson, and S. Kröll, Initial experiments concerning quantum information processing in rare-earth-ion doped crystals, Phys. Scr. T102, 178 (2002).

[20] J. Wesenberg and K. Mølmer, Robust quantum gates and a bus architecture for quantum computing with rare-earth-ion-doped crystals, Phys. Rev. A 68, 012320 (2003).

[21] J. H. Wesenberg, K. Mølmer, L. Rippe, and S. Kröll, Scalable designs for quantum computing with rare-earth-ion-doped crystals, Phys. Rev. A 75, 012304 (2007).

[22] A. Walther, B. Julsgaard, L. Rippe, Y. Ying, S. Kröll, R. Fisher, and S. Glaser, Extracting high fidelity quantum computer hardware from random systems, Phys. Scr. T137, 014009 (2009).

[23] A. Walther, L. Rippe, Y. Yan, J. Karlsson, D. Serrano, A. N. Nilsson, S. Bengtsson, and S. Kröll, High-fidelity readout scheme for rare-earth solid-state quantum computing, Phys. Rev. A 92, 022319 (2015).

[24] R. L. Ahlefeldt, M. J. Pearce, M. R. Hush, and M. J. Sellars, Quantum processing with ensembles of rare-earth ions in a stoichiometric crystal, Phys. Rev. A 101, 012309 (2020).

[25] M. Grimm, A. Beckert, G. Aeppli, and M. Müller, Universal quantum computing using electronuclear wavefunctions of rareearth ions, PRX Quantum 2, 010312 (2021).

[26] V. Hizhnyakov, V. Boltrushko, H. Kaasik, and Y. Orlovskii, Rare earth ions doped mixed crystals for fast quantum computers with optical frequency qubits, Opt. Commun. 485, 126693 (2021).

[27] A. Kinos, D. Hunger, R. Kolesov, K. Mølmer, H. de Riedmatten, P. Goldner, A. Tallaire, L. Morvan, P. Berger, S. Welinski, K. Karrai, L. Rippe, S. Kröll, and A. Walther, Roadmap for rare-earth quantum computing, arXiv:2103.15743.

[28] A. Kinos, L. Rippe, S. Kröll, and A. Walther, Designing gate operations for single-ion quantum computing in rare-earth-iondoped crystals, Phys. Rev. A 104, 052624 (2021).

[29] F. Könz, Y. Sun, C. W. Thiel, R. L. Cone, R. W. Equall, R. L. Hutcheson, and R. M. Macfarlane, Temperature and concentration dependence of optical dephasing, spectral-hole lifetime, and anisotropic absorption in $\mathrm{Eu}^{3+}: \mathrm{Y}_{2} \mathrm{SiO}_{5}$, Phys. Rev. B 68 , 085109 (2003).

[30] M. Nilsson, L. Rippe, S. Kröll, R. Klieber, and D. Suter, Hole-burning techniques for isolation and study of individual hyperfine transitions in inhomogeneously broadened solids demonstrated in $\operatorname{Pr}^{3+}: \mathrm{Y}_{2} \mathrm{SiO}_{5}$, Phys. Rev. B 70, 214116 (2004).

[31] L. Rippe, M. Nilsson, S. Kröll, R. Klieber, and D. Suter, Experimental demonstration of efficient and selective population transfer and qubit distillation in a rare-earth-metal-ion-doped crystal, Phys. Rev. A 71, 062328 (2005). 
[32] B. Lauritzen, N. Timoney, N. Gisin, M. Afzelius, H. de Riedmatten, Y. Sun, R. M. Macfarlane, and R. L. Cone, Spectroscopic investigations of $\mathrm{Eu}^{3+}: \mathrm{Y}_{2} \mathrm{SiO}_{5}$ for quantum memory applications, Phys. Rev. B 85, 115111 (2012).

[33] A. Kinos, L. Rippe, A. Walther, and S. Kröll, Microscopic treatment of instantaneous spectral diffusion and its effect on quantum gate fidelities in rare-earth-doped crystals, arXiv:2110.09884 [Phys. Rev. A (to be published)].

[34] T. Utikal, E. Eichhammer, L. Petersen, A. Renn, S. Goetzinger, and V. Sandoghdar, Spectroscopic detection and state preparation of a single praseodymium ion in a crystal, Nat. Commun. 5, 3627 (2014).

[35] K. Xia, R. Kolesov, Y. Wang, P. Siyushev, R. Reuter, T. Kornher, N. Kukharchyk, A. D. Wieck, B. Villa, S. Yang, and J. Wrachtrup, All-Optical Preparation of Coherent Dark States of a Single Rare Earth Ion Spin in a Crystal, Phys. Rev. Lett. 115, 093602 (2015).

[36] R. Kolesov, S. Lasse, C. Rothfuchs, A. D. Wieck, K. Xia, T. Kornher, and J. Wrachtrup, Superresolution Microscopy of Single Rare-Earth Emitters in YAG and H3 Centers in Diamond, Phys. Rev. Lett. 120, 033903 (2018).

[37] T. Zhong, J. M. Kindem, J. G. Bartholomew, J. Rochman, I. Craiciu, V. Verma, S. W. Nam, F. Marsili, M. D. Shaw, A. D. Beyer, and A. Faraon, Optically Addressing Single Rare-Earth Ions in a Nanophotonic Cavity, Phys. Rev. Lett. 121, 183603 (2018).

[38] M. Raha, S. T. Chen, C. M. Phenicie, S. Ourari, A. M. Dibos, and J. D. Thompson, Optical quantum nondemolition measurement of a single rare earth ion qubit, Nat. Commun. 11, 1605 (2020).

[39] J. M. Kindem, A. Ruskuc, J. G. Bartholomew, J. Rochman, Y. Q. Huan, and A. Faraon, Control and single-shot readout of an ion embedded in a nanophotonic cavity, Nature (London) 580, 201 (2020).

[40] J. J. Longdell and M. J. Sellars, Experimental demonstration of quantum-state tomography and qubit-qubit interactions for rare-earth-metal-ion-based solid-state qubits, Phys. Rev. A 69, 032307 (2004).

[41] J. J. Longdell, M. J. Sellars, and N. B. Manson, Demonstration of Conditional Quantum Phase Shift Between Ions in a Solid, Phys. Rev. Lett. 93, 130503 (2004).

[42] R. L. Ahlefeldt, D. L. McAuslan, J. J. Longdell, N. B. Manson, and M. J. Sellars, Precision Measurement of Electronic Ion-Ion Interactions between Neighboring $\mathrm{Eu}^{3+}$ Optical Centers, Phys. Rev. Lett. 111, 240501 (2013).

[43] J. Jackson, Classical Electrodynamics, 3rd ed. (John Wiley \& Sons, Inc., New York, 1998).

[44] R. Raussendorf and J. Harrington, Fault-Tolerant Quantum Computation with High Threshold in Two Dimensions, Phys. Rev. Lett. 98, 190504 (2007).

[45] A. G. Fowler, A. M. Stephens, and P. Groszkowski, Highthreshold universal quantum computation on the surface code, Phys. Rev. A 80, 052312 (2009).

[46] A. M. Steane, Overhead and noise threshold of faulttolerant quantum error correction, Phys. Rev. A 68, 042322 (2003).
[47] B. Maksimov, V. Ilyukhin, Y. Kharitonov, and N. Belov, Crystal structure of yttrium oxyorthosilicate $\mathrm{Y}_{2} \mathrm{O}_{3} \cdot \mathrm{SiO}_{2}=\mathrm{Y}_{2} \mathrm{SiO}_{5}$ - dual function of yttrium, Sov. Phys. Crystallogr. 15, 806 (1971).

[48] S. Bengtsson, Simulation and modeling of rare earth ion based quantum gate operations, Master's thesis, Lund University, 2012.

[49] $\mathrm{Y}_{2} \mathrm{SiO}_{5}\left(\mathrm{Y}_{2}\left[\mathrm{SiO}_{4}\right] \mathrm{O}\right.$ ht $)$ crystal structure: Datasheet from "Pauling file multinaries edition - 2012" in Springer Materials (2021), copyright 2016 Springer-Verlag Berlin Heidelberg \& Material Phases Data System (MPDS), Switzerland \& National Institute for Materials Science (NIMS), Japan, https://materials. springer.com/isp/crystallographic/docs/sd_1801962.

[50] F. R. Graf, A. Renn, G. Zumofen, and U. P. Wild, Photon-echo attenuation by dynamical processes in rare-earth-ion-doped crystals, Phys. Rev. B 58, 5462 (1998).

[51] G. D. Mahan, Local-field corrections to Coulomb interactions, Phys. Rev. 153, 983 (1967).

[52] T. Christiansson, A first step towards quantum computing in rare-earth-ion-doped crystals, Master's thesis, Lund University, Lund Reports in Atomic Physics, LRAP-266, 2001.

[53] A. Mock, R. Korlacki, S. Knight, and M. Schubert, Anisotropy and phonon modes from analysis of the dielectric function tensor and the inverse dielectric function tensor of monoclinic yttrium orthosilicate, Phys. Rev. B 97, 165203 (2018).

[54] A. M. Stoneham, Shapes of inhomogeneously broadened resonance lines in solids, Rev. Mod. Phys. 41, 82 (1969).

[55] H. Yamamoto, Y. Otomo, and T. Kano, Satellite lines due to ion-pairs in luminescence spectra of $\mathrm{Y}_{2} \mathrm{O}_{2} \mathrm{~S}-\mathrm{Eu}^{3+}$, J. Phys. Soc. Jpn. 26, 137 (1969).

[56] M. J. Sellars, E. Fraval, and J. J. Longdell, Investigation of static electric dipole-dipole coupling induced optical inhomogeneous broadening in $\mathrm{Eu}^{3+}: \mathrm{Y}_{2} \mathrm{SiO}_{5}$, J. Lumin. 107, 150 (2004).

[57] R. W. Equall, Y. Sun, R. L. Cone, and R. M. Macfarlane, Ultraslow Optical Dephasing in $\mathrm{Eu}^{3+}: \mathrm{Y}_{2} \mathrm{SiO}_{5}$, Phys. Rev. Lett. 72, 2179 (1994).

[58] T. Böttger, C. W. Thiel, R. L. Cone, and Y. Sun, Controlled compositional disorder in $\mathrm{Er}^{3+}: \mathrm{Y}_{2} \mathrm{SiO}_{5}$ provides a wide-bandwidth spectral hole burning material at $1.5 \mu \mathrm{m}$, Phys. Rev. B 77, 155125 (2008).

[59] K. Debnath, A. H. Kiilerich, and K. Mølmer, Ancilla-mediated qubit readout and heralded entanglement between rare-earth dopant ions in crystals, Phys. Rev. A 103, 043705 (2021).

[60] T. Förster, Zwischenmolekulare energiewanderung und fluoreszenz, Ann. Phys. 437, 55 (1948).

[61] D. L. Dexter, A theory of sensitized luminescence in solids, J. Chem. Phys. 21, 836 (1953).

[62] D. Serrano, Y. Yan, J. Karlsson, L. Rippe, A. Walther, S. Kröll, A. Ferrier, and P. Goldner, Impact of the ion-ion energy transfer on quantum computing schemes in rare-earth doped solids, J. Lumin. 151, 93 (2014).

[63] J. A. Caird, A. J. Ramponi, and P. R. Staver, Quantum efficiency and excited-state relaxation dynamics in neodymium-doped phosphate laser glasses, J. Opt. Soc. Am. B 8, 1391 (1991).

[64] R. Beach, M. D. Shinn, L. Davis, R. W. Solarz, and W. F. Krupke, Optical-absorption and stimulated-emission of neodymium in yttrium orthosilicate, IEEE J. Quantum Electron. 26, 1405 (1990). 
[65] S. Huang, Development of a temperature control system for spectroscopic measurements with rare-earth doped crystals, Master's thesis, Lund University, 2014.

[66] M. Inokuti and F. Hirayama, Influence of energy transfer by the exchange mechanism on donor luminescence, J. Chem. Phys. 43, 1978 (1965).
[67] C. Brecher, Europium in the ultraphosphate lattice: Polarized spectra and structure of $\mathrm{EuP}_{5} \mathrm{O}_{14}$, J. Chem. Phys. 61, 2297 (1974).

[68] R. L. Ahlefeldt, N. B. Manson, and M. J. Sellars, Optical lifetime and linewidth studies of the ${ }^{7} \mathrm{~F}_{0} \rightarrow{ }^{5} \mathrm{D}_{0}$ transition in $\mathrm{EuCl}_{3} \cdot 6 \mathrm{H}_{2} \mathrm{O}$ : A potential material for quantum memory applications, J. Lumin. 133, 152 (2013). 